Journal Club

Editor's Note: These short, critical reviews of recent papers in the Journal, written exclusively by graduate students or postdoctoral fellows, are intended to summarize the important findings of the paper and provide additional insight and commentary. For more information on the format and purpose of the Journal Club, please see http://www.jneurosci.org/misc/ifa_features.shtml.

\title{
Homeostatic Plasticity of Neurotransmitter Release Probability Is Determined by Presynaptic Calcium Influx
}

\author{
Jaichandar Subramanian \\ Unit on Behavioral Genetics, Laboratory of Molecular Pathophysiology, National Institute of Mental Health, National Institutes of Health, Bethesda, \\ Maryland 20892
}

Review of Zhao et al.

Information processing and plasticity in neural circuits rely on the proper balance of excitatory and inhibitory signals. Changes in the excitability of neurons or synaptic strength are compensated either locally near the altered synapses or globally at all synapses to maintain circuit stability, a process defined as homeostatic plasticity (Turrigiano, 2011). Homeostatic plasticity has been observed both in vitro and in vivo in different model systems, including flies and mammals (Turrigiano, 2011).

For mammalian fast central synapses, the cellular and molecular mechanisms of homeostatic plasticity have been determined primarily from studies on dissociated cortical and hippocampal neurons in culture (Thiagarajan et al., 2007). A general method to study homeostatic plasticity in dissociated cultures is to globally perturb neuronal excitability by bath application of pharmacological reagents that decrease or increase neuronal activity. In general, these studies have found that inactivity of neurons enhances synaptic strength, whereas hyperactivity reduces it through both structural and functional modification of synapses (Thiagarajan et

\footnotetext{
Received June 1, 2011; revised July 1, 2011; accepted July 1, 2011.

This work was supported by the Intramural Research Program of National Institute for Mental Health. I thank Dr. Alexei Morozov and Aarthi Narayan for critical reading of this manuscript.

Correspondence should be addressed to Jaichandar Subramanian, Unit on Behavioral Genetics, Laboratory of Molecular Pathophysiology, National Institute of Mental Health, National Institutes of Health, Bethesda, MD 20892. E-mail: jaichandar@gmail.com.

DOI:10.1523/JNEUROSCI.2734-11.2011

Copyright $\odot 2011$ the authors $\quad$ 0270-6474/11/3111097-03\$15.00/0
}

al., 2007). Postsynaptic modifications include changes in the size of the postsynaptic density and the number of AMPA receptors. Presynaptic changes involve alteration of the size of boutons and active zones and of the probability of neurotransmitter release (Thiagarajan et al., 2007).

The mechanism underlying homeostatic regulation of neurotransmitter release probability remains largely unexplored. Release probability is regulated by multiple factors such as the size of the readily releasable pool of vesicles, the level of calcium influx, and the responsiveness of release machinery to calcium. Although some studies have reported homeostatic regulation of readily releasable pool size, the magnitude of this effect is not enough to fully explain the observed homeostatic change in release probability (Thiagarajan et al., 2007). Which other factors that regulate release probability are also under homeostatic control remain to be tested.

Measuring calcium influx in presynaptic terminals of central mammalian synapses is challenging. Calcium influx is generally measured using electrophysiological or optical approaches, but the small diameter of axons of most central neurons precludes electrophysiological recordings. The optical approach has mostly been limited to using calcium indicator dyes. However, noninvasive bulk loading of calcium indicator dyes uniformly labels all neurites from every cell, making it difficult to unambiguously de- tect axons from an individual cell. Recent developments in genetically encoded calcium indicators (GECIs) have overcome these limitations. When introduced by low-efficiency chemical transfection procedures, individual neurons with clearly resolved neurites containing GECIs can be labeled. However, most GECIs indiscriminately label all parts of cells and are not specific to presynaptic terminals.

In a recent paper published in The Journal of Neuroscience, Zhao et al. (2011) show that presynaptic calcium influx is homeostatically regulated and that this regulation underlies homeostatic plasticity of neurotransmitter release probability. To overcome the aforementioned technical limitations, Zhao et al. (2011) used a GECI, SyGCaMP2 (Dreosti et al., 2009), which localizes to presynaptic terminals. SyGCaMP2 is made by fusing GCaMP2, a GFP-based calcium sensor, to the $\mathrm{C}$ terminus of synaptophysin. Fusion to synaptophysin confers presynaptic localization to GCaMP2 (Dreosti et al., 2009).

To study homeostatic regulation of calcium influx in presynaptic terminals, Zhao et al. (2011) used $\sim 2$-week-old cultures of dissociated hippocampal neurons expressing SyGCaMP2. One to two days before imaging, sister cultures were either left untreated (control) or treated with TTX, a sodium channel blocker that suppresses network activity, or gabazine, a $\mathrm{GABA}_{\mathrm{A}}$ receptor blocker that enhances network activity. SyGCaMP2 response 
$\left(\Delta F / F_{0}\right)$ to field electrical stimulation (1ms pulses at $20 \mathrm{~Hz}$ ) was imaged for a variable number of action potentials (APs) for each treatment group. Although the SyGCaMP2 response was highly variable between boutons (Zhao et al., their Fig. $2 A, B)$, spatial averaging of responses from multiple boutons produced a reliable response with high signal-to-noise ratio, even for single AP stimulation (Zhao et al., their Fig. 2C). The SyGCaMP2 response was linear up to at least 5 APs (Zhao et al., their Fig. 2D). Compared to control conditions, the slope of the response in TTX- and gabazinetreated cultures were 33\% higher and 23\% lower, respectively (Zhao et al., their Fig. $2 D$ ), indicating that calcium influx into presynaptic terminals is homeostatically regulated.

To test whether homeostatic mechanisms of presynaptic calcium influx and neurotransmitter release probability are related, Zhao et al. (2011) sought to determine homeostatic changes in neurotransmitter release in their system. Neurotransmitter release is commonly measured by electrophysiological recordings from postsynaptic neurons or optical imaging of FM dye uptake in presynaptic terminals. Although physiological recordings of evoked neurotransmitter release necessitate ruling out the postsynaptic effect of network manipulation, FM dye techniques are not sensitive enough to detect responses to single APs. As an alternative method, GFP-based probes to monitor synaptic vesicle exocytosis have been developed. These probes contain a $\mathrm{pH}$ sensitive GFP ( $\mathrm{pHluorin}$ ) fused to the lumenal domain of synaptic vesicle proteins. As the vesicles exocytose, the pHluorin moiety previously exposed to the acidic $\mathrm{pH}$ of the vesicle lumen gets exposed to the neutral $\mathrm{pH}$ on the extracellular side, resulting in an increase in fluorescence of 20 -fold per vesicle (Burrone et al., 2006).

Zhao et al. (2011) used SypHy (pHluorin tagged to synaptophysin), which is sensitive enough to detect vesicle exocytosis following a single AP (after averaging) (Granseth et al., 2006). To evoke neurotransmitter release, Zhao et al. (2011) delivered 10 pulses of single APs [average SypHy response $\left(\Delta F / F_{0}\right)$ of the 10 pulses determines the response to a single AP], followed by $40 \mathrm{APs}$ at $20 \mathrm{~Hz}$ (which has been shown to cause exocytosis of the entire readily releasable pool). The ratio between the SypHy response to a single AP and the response to $40 \mathrm{APs}$ corresponds to the release probability. As in homeostatic regulation of neurotransmitter release, Zhao et al. (2011) found that the response to a single AP was increased in cultures treated with TTX and reduced in cultures treated with gabazine compared to the control (Zhao et al., their Fig. 3B). On average, TTX-treated cells exhibited a threefold higher SyPhy response than gabazine-treated cells. Interestingly, Zhao et al. (2011) found no or little change in the response to 40 APs following TTX or gabazine treatment, respectively (Zhao et al., their Fig. 3B), suggesting that the size of the readily releasable pool of vesicles is not homeostatically regulated.

Because Zhao et al. (2011) found no homeostatic effect on the readily releasable pool, they next investigated whether the observed homeostatic regulation of calcium influx was responsible for the homeostatic regulation of release probability. If these two processes are related, then the direction of homeostatic changes in individual boutons for these two processes will also be similar. Ideally, this can be tested by simultaneously measuring calcium influx and release probability in the same boutons before and after activity manipulations; but lacking tools with the required sensitivity, Zhao et al. (2011) compared the distribution of SyGCaMP2 responses and release probability obtained from different set of boutons. Both responses were highly variable between boutons (Zhao et al., their Figs. $2 A$ and $3 A$ ) and could be described as a gamma distribution (Zhao et al., their Fig. 3C,D). Furthermore, for both responses, the spread of the distribution (as defined by the scale parameter $\beta$ ) was significantly lower in gabazinetreated cultures and higher in TTXtreated cultures compared to the control. This parallel in the two responses is consistent with the homeostatic regulation of calcium influx and release probability being related.

It has been proposed that homeostatic modifications in response to global perturbation of network activity act equally on all synapses to retain the relative strength of synapses (Turrigiano, 2011). If this is true, one would expect TTX-treated and gabazine-treated cultures to exhibit similar spreads of distribution of responses, albeit at the opposite ends of the response scale. However, the broad and narrow distribution of responses in TTX-treated and gabazine-treated cultures, respectively, suggest that high-responding boutons are capable of further increases in responses after TTX treatment, whereas low-responding boutons cannot further reduce responses following gabazine treatment. One possible explanation for this result is that the activitydriven, calcium influx-dependent internal- ization of calcium channels (Green et al., 2007) is more likely to occur in boutons that have high initial response than in boutons with weak initial response. In light of the results by Zhao et al. (2011), the idea of uniform change in synaptic response to network activity may need to be reexamined. Chronic imaging of the same boutons before and after treatment could address this issue.

Although the observed results thus far rule out a homeostatic mechanism regulating the readily releasable pool size and support a role for homeostatic regulation of calcium influx, a third mechanism for homeostatic regulation exists, namely homeostatic regulation of the efficiency of release machinery. Zhao et al. (2011) used a theoretical approach to rule out such a mechanism. To do this, they compared the relationship between release probability and SyGCaMP2 response under each treatment condition (Zhao et al., their Fig. 4). For each condition, they averaged the responses from all boutons. For an approximately twofold increase in SyGCaMP2 response per AP in TTX-treated cells compared to gabazine-treated cells, the release probability increased approximately eightfold (Zhao et al., their Fig. 4), indicating a third-power relationship between SyGCaMP2 response and neurotransmitter release. The twofold increase in SyGCaMP2 response is well within the linear range of this probe and can be directly equated to increases in calcium influx. Since the network activity manipulations did not alter the known cooperative relationship between calcium influx and neurotransmitter release, Zhao et al. (2011) argue that the homeostatic plasticity of release probability is primarily determined by changes in calcium influx.

While the evidence for the relationship between homeostatic changes in calcium influx and release probability are compelling, it is unclear how Zhao et al. (2011) reconcile their findings with previously published data that show a role for the readily releasable pool size and vesicle priming in presynaptic homeostatic plasticity (Moulder et al., 2006; Thiagarajan et al., 2007). Nonetheless, the findings of Zhao et al. (2011) and their technical tools have opened the possibility of identifying the nature of the presynaptic ion channels involved in homeostatic plasticity and how these channels are modified in response to network activity. Such mechanistic understanding is necessary to develop tools to control homeostatic synaptic plasticity, the failure of which has been associated with neurological disorders such as 
epilepsy, autism, and schizophrenia (Turrigiano, 2011).

\section{References}

Burrone J, Li Z, Murthy VN (2006) Studying vesicle cycling in presynaptic terminals using the genetically encoded probe synaptopHluorin. Nat Protoc 1:2970-2978.

Dreosti E, Odermatt B, Dorostkar MM, Lagnado L (2009) A genetically encoded reporter of synaptic activity in vivo. Nat Methods 6:883-889.
Granseth B, Odermatt B, Royle SJ, Lagnado L (2006) Clathrin-mediated endocytosis is the dominant mechanism of vesicle retrieval at hippocampal synapses. Neuron 51:773-786.

Green EM, Barrett CF, Bultynck G, Shamah SM, Dolmetsch RE (2007) The tumor suppressor eIF3e mediates calcium-dependent internalization of the L-type calcium channel CaV1.2. Neuron 55:615-632.

Moulder KL, Jiang X, Taylor AA, Olney JW, Mennerick S (2006) Physiological activity depresses synaptic function through an ef- fect on vesicle priming. J Neurosci 26: $6618-6626$.

Thiagarajan TC, Lindskog M, Malgaroli A, Tsien RW (2007) LTP and adaptation to inactivity: overlapping mechanisms and implications for metaplasticity. Neuropharmacology 52:156-175.

Turrigiano G (2011) Too many cooks? Intrinsic and synaptic homeostatic mechanisms in cortical circuit refinement. Annu Rev Neurosci 34:89-103.

Zhao C, Dreosti E, Lagnado L (2011) Homeostatic synaptic plasticity through changes in presynaptic calcium influx. J Neurosci 31:7492-7496. 